\title{
Measurement of Thermal Diffusivity by Lock-in Thermography
}

\author{
by P.G. Bison, S. Marinetti, E. Grinzato
}

CNR-ITEF, C.so Stati Uniti 4, 35127 Padova, Italy, E-mail: paolo.bison@itef.pd.cnr.it

\begin{abstract}
Diffusivity is generally measured on small sample under laboratory conditions where each side of the specimen is accessible, but one-side measurement may be necessary in such applications requiring in situ inspection. Experimental results are reported applying a thermoelectric unit in contact with the sample, acting alternatively as heat source and sink and generating thermal waves on the specimen. The read out of temperature is done on the same side, laterally to the source. This allows estimating the diffusivity of the material along the stimulated surface from the peaks displacement velocity or waves phase shifting measurement. Simple formulas coming from an adiabatic model furnish diffusivity values that overestimate the real one with the increase of the harmonic stimulus period. A procedure to extract the real value of diffusivity is proposed.
\end{abstract}

\section{Introduction}

Up today the most used method for Thermal Diffusivity measurement through thermography is made according to the so called Flash Method that works in transmission mode (also referred as two side measurement). Generally speaking, diffusivity is measured on small sample under laboratory conditions. Reflection mode (one side) measurement can be performed through photothermal techniques that require a laser source and are quite difficult to be performed outside of a lab. On the other hand, one side measurement may be necessary in such applications requiring in situ measurement.

The proposed idea is to built up a device that is capable of generating, on the surface of the material, thermal waves having a null net heat flux and to detect them by thermography. That was done keeping a thermoelectric unit in contact with the material surface and driving it in successive heating and cooling stages. When the harmonic temperature regime is reached on the surface one can measure the velocity of the sinusoidal peak propagation in space or the phase shift of the thermal wave. According to simple formulas coming from an adiabatic analytical model one can extract the diffusivity value from the velocity/phase information.

Previous works showed that the diffusivity estimation from this non-adiabatic process suffers for the adiabatic formulation adopted. In fact, a systematic increase of the diffusivity values was noticed when the stimulating flux period was increased. The aim of this work is to establish a simple but effective rule to extract the correct diffusivity value, getting rid of the trend in the raw diffusivity estimation.

A mathematical simulation of the thermal process is a tool for a deeper investigation of the testing techniques in order to apply it in situ. For instance the influence of heat exchange with the environment and finite size of the sample have been taken into account.

An important advantage of this technique is offered by the short time necessary to reach the permanent regime and avoiding the sample overheating. 


\section{Theoretical model}

The theoretical model describing the test is shown in Fig. 1. The part of the specimen beneath the thermoelectric unit is subjected to a periodic stimulus and the temperature is observed on the adjacent free surface by a thermographic camera. In the case of thermally thin sample the temperature gradient between the stimulated side of the sample and the back one is very low. In such a way the periodic thermal flux propagates as a plane wave, laterally to the heated zone.

To estimate the thermal diffusivity from the temperature data, the model used is the analytical solution of the Fourier equation for 1D heat diffusion, inside a semi-infinite solid subjected to a periodic heating/cooling flux $F=F_{0} \cos (\omega t)$. The propagation along the $x$-axis, in adiabatic conditions, is governed by the following equation [1, 2]:

$$
T(t, x)=\frac{F_{0}}{\sqrt{2} \cdot \lambda \cdot k} \cdot e^{-k x} \cos \left(\omega t-k x-\frac{\pi}{4}\right)
$$

where $T$ is temperature, $t$ is time, $\alpha$ is the diffusivity, $\lambda$ conductivity, $\mathrm{k}=(\omega / 2 \alpha)^{1 / 2}$ and $\omega=2 \pi / t_{0}$ with $t_{0}$ the oscillation period.

Knowing the oscillation period $t_{0}$ and measuring the velocity $v$ at which the waves move inside the solid, it is possible to recover the thermal diffusivity:

$$
\alpha=\frac{t_{0}}{4 \pi} \cdot v^{2}
$$

Another way to recover the value of $\alpha$ is computing the slope of the phase $k=d \phi / d x$ and applying the following relation:

$$
\alpha=\frac{\pi}{t_{0}} \cdot\left(\frac{d x}{d \phi}\right)^{2}
$$

These simple formulas are not unconditionally applicable to the experimental data coming from the layout depicted in Fig. 1, but they work when the thermal diffusion length $L=1 / \mathrm{k}$ in Eq. (1) is much greater than the specimen thickness $d$ [3]. This gives the constraint on the oscillation period $t_{0}$ :

$$
t_{0}>>\frac{\pi \cdot d^{2}}{\alpha}
$$

As reported in previous works [4], when formulae (2) and (3) are applied to experimental data acquired in presence of convective exchange with the environment, the diffusivity value is overestimated and it increases with the period. Numerical simulations (based on FEM) showed that, once condition (4) is met, for small Biot number $(B i=h d / \lambda)$ the $\alpha$ vs $t_{0}$ function could be approximate with a straight line whose intercept is a good estimate of the diffusivity value. Fig.2 shows the simulation result relative to a stainless steel specimen (AISI 304 alloy): the intercept value is $3.8610^{-6} \mathrm{~m}^{2} \mathrm{~s}^{-1}$ while the diffusivity value was set to $3.9510^{-6} \mathrm{~m}^{2} \mathrm{~s}^{-1}$ (relative error, 2.3 $\%)$. 


\section{Experimental set up}

The experimental set up is shown in Fig. 3. The apparatus is composed of a thermoelectric device, based on the Peltier effect, acting as heat source and sink according to the electrical current sign that flows through it. This unit is characterized by a thermal power of $50 \mathrm{~W}$ with a maximum electrical power of 90 VA. The device is driven by a power supply unit remotely controlled by a computer that provides the suitable rectified waveform. A switch box exchange the polarity making the thermoelectric unit working in heating and cooling stage alternatively. The thermoelectric unit is cooled by a heat exchanger fed by tap water at constant temperature. The thermographic camera records the surface temperature aside of the stimulated zone. A special ruler is included in the field of view in order to recover the pixel dimension that is a crucial measurement in the diffusivity estimate.

\section{Experimental result}

Three slabs of different materials were tested: a) inconel, $1.3 \mathrm{~mm}$ thick (diffusivity nominal values from literature ranging around $3.1 \div 3.4^{*} 10^{-6}$ ), b) aisi-304, $2 \mathrm{~mm}$ thick (nominal values from literature ranging around $3.95 \div 4.1^{*} 10^{-6}$ ), c) PVC, $1.5 \mathrm{~mm}$ thick (nominal values from literature ranging around $1.1 \div 2.0^{*} 10^{-7}$ ). Tests were repeated for each material at different harmonic stimulus. Periods ranging from 60 to $130 \mathrm{~s}$ for aisi-304, from 60 to $120 \mathrm{~s}$ for inconel and from 60 to $300 \mathrm{~s}$ for PVC were used.

An example of the data collected in one test is resumed in fig. 4a,b,c where the thermal wave propagation inside the material is visualized for the slab of PVC tested at $150 \mathrm{~s}$ period. In fig. $4 \mathrm{a}$ the temperature grabbed along the propagation line on the material surface is collected at successive time and stacked in the image. Fig. 4b shows some sampled columns of the previous image at increasing space position (from the heating/cooling source). Here sinusoidal curves dumped and phase shifted are depicted. Fig. 4c shows some sampled rows at increasing time values and therefore the space propagation and dumping at different time is visualized.

From the data collected and organized as shown before it is possible to extract the information relative to the velocity of peaks (maximum or minimum) propagation as shown in fig. $5 \mathrm{a}$, or the phase of the propagated wave as a function of space as in fig. 5b. Diffusivity is immediately related to those measured quantities through eq. 2 and/or 3 .

Results from our measurements are summarized in fig. 6. Inconel and aisi-304 refers to the left axis values while PVC is referred to the right one. It must be stressed the increase of the apparent diffusivity value vs. the increase of the period of the heating/cooling process, confirming experimentally what were depicted from the numerical simulation. As mentioned before, the intercept of the straight line is a good approximation of the true diffusivity.

Final diffusivity results for the three materials are: a) $\alpha=3.98^{\star} 10^{-6}$ for aisi-304; b) $\alpha=3.53^{\star} 10^{-6}$ for inconel; c) $\alpha=1.68^{*} 10^{-7}$ for PVC. No alternative measurements of diffusivity were possible until now to confirm the reliability of the results of this method but values are in good agreement with the literature. Notice also that results show a very little dispersion among different experiments. On the other hand, the geometrical calibration of distances on thermograms is very critical and not trivial to be correctly evaluated. This uncertainty is important because this quantity appears with the square power in relations $(2,3)$, giving a systematic deviation from the real value. 


\section{Conclusion}

The Lock-in method to measure diffusivity by a Peltier cell was applied to thermally thin samples of high and low diffusivity materials. From a simple analytical model (1D) an inversion procedure was derived and applied with good results. Significant improvements of the stimulating equipment and about the presence of heat convection on the surface are presented. For the near future new algorithms for the inversion are in the testing phase.

\section{References}

1. H.S. Carslaw, J.C. Jaeger, Conduction of heat in solids, Oxford Unversity Press, London, 1959.

2. P.G. Bison, A. Muscio, E. Grinzato, "Thermal parameters estimation by heating and cooling and thermographic measurement" Proceedings of SPIE Conference Thermosense XXI, vol. 3700, Orlando, Florida, April 1999.

3. S. Marinetti, P.G. Bison, E. Grinzato, A. Muscio, "Thermal diffusivity measurement of stainless steel by periodic heating technique" Proceedings of $5^{\text {th }}$ AITA Workshop, Venezia September 2930, to be published.

4. G.S. Barozzi, M.A. Corticelli, A. Muscio, P. Tartarini, "Numerical investigation of a one-side measurement technique for thermal diffusivity" $17^{\text {th }}$ National Heat Transfer Conference of the UIT, Ferrara, Italy, pp. 421-434, 1999.

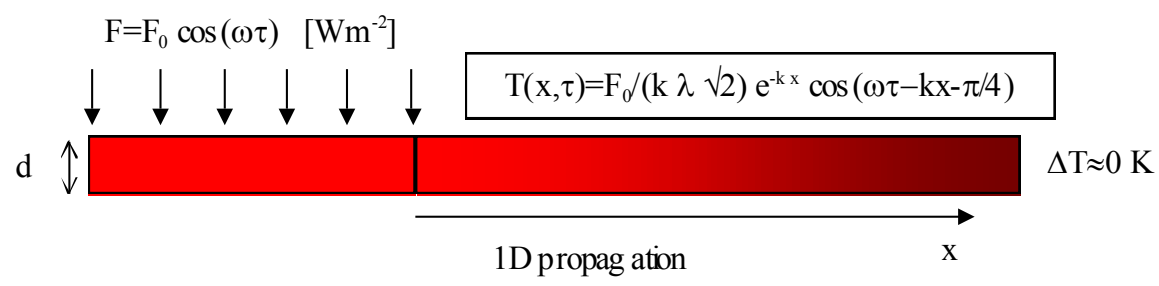

Figure 1. Thermal model of a thermally thin slab submitted to thermal waves

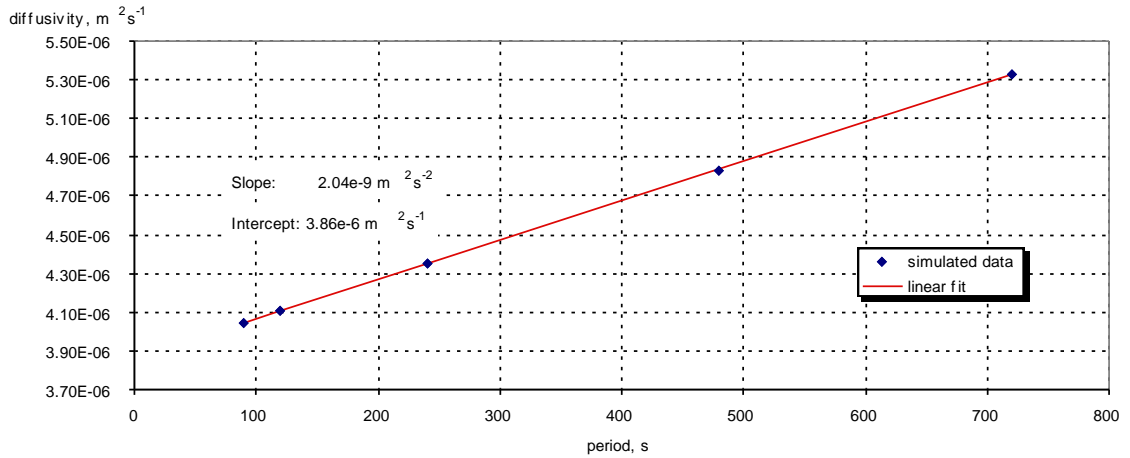

Figure 2: Diffusivity overestimate due to convective heat exchange neglecting (FEM simulation of AISI 304) 
http://dx.doi.org/10.21611/qirt.2000.008

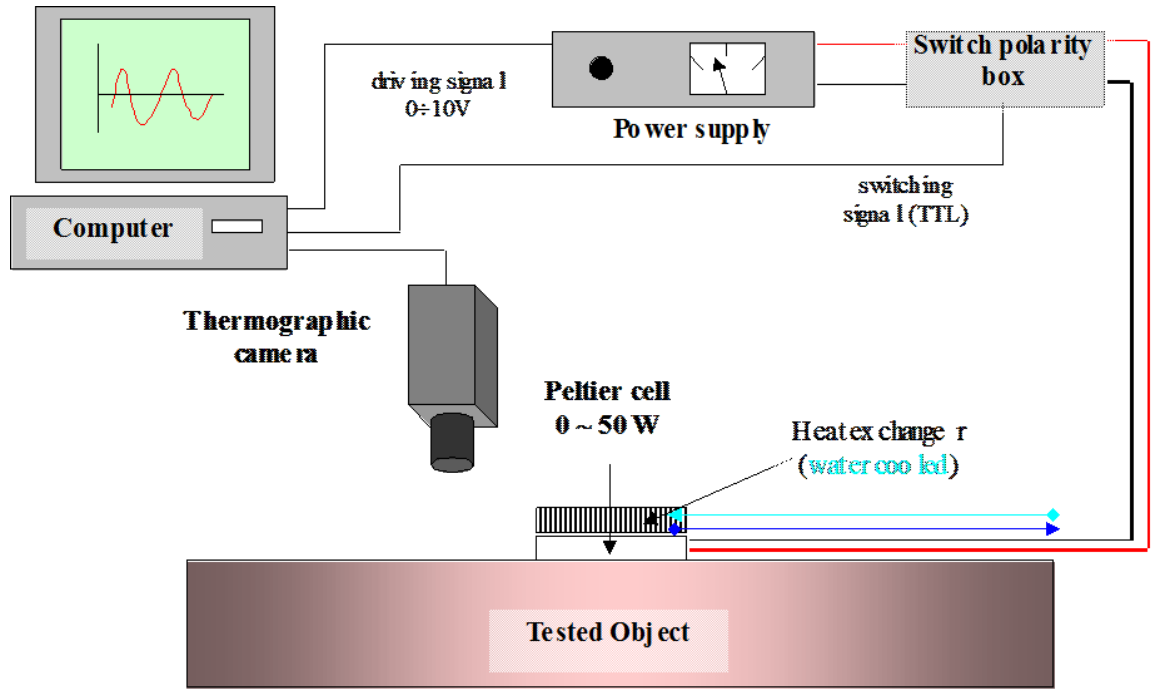

Figure 3. Experimental set up for the Lock-in Diffusivity (LiD) measurement

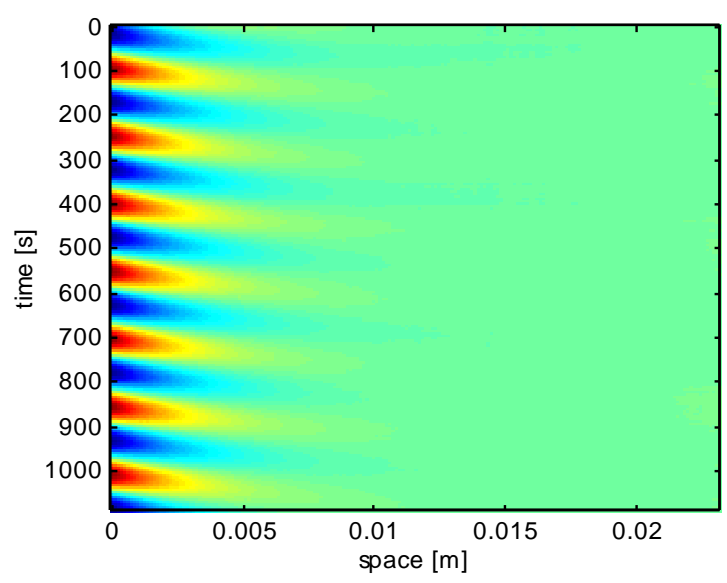

Figure 4a. Thermal Wave Visualization (spacetime map) for PVC $1.5 \mathrm{~mm}$ thick, period $\cong 150 \mathrm{~s}$ for the applied harmonic heat flux

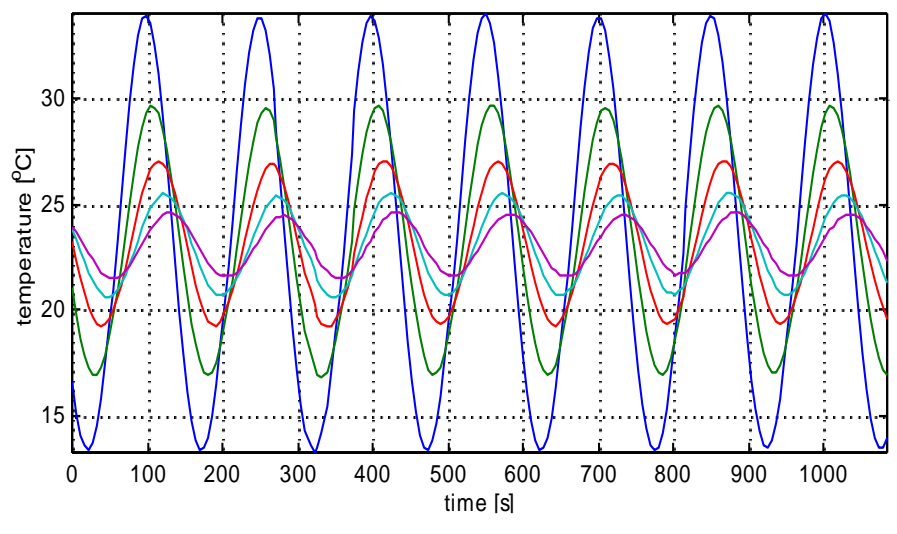

Figure 4b. Thermal Wave phase shifting and dumping (curves sampled at increasing space position) for PVC $1.5 \mathrm{~mm}$ thick, period $\cong 150 \mathrm{~s}$

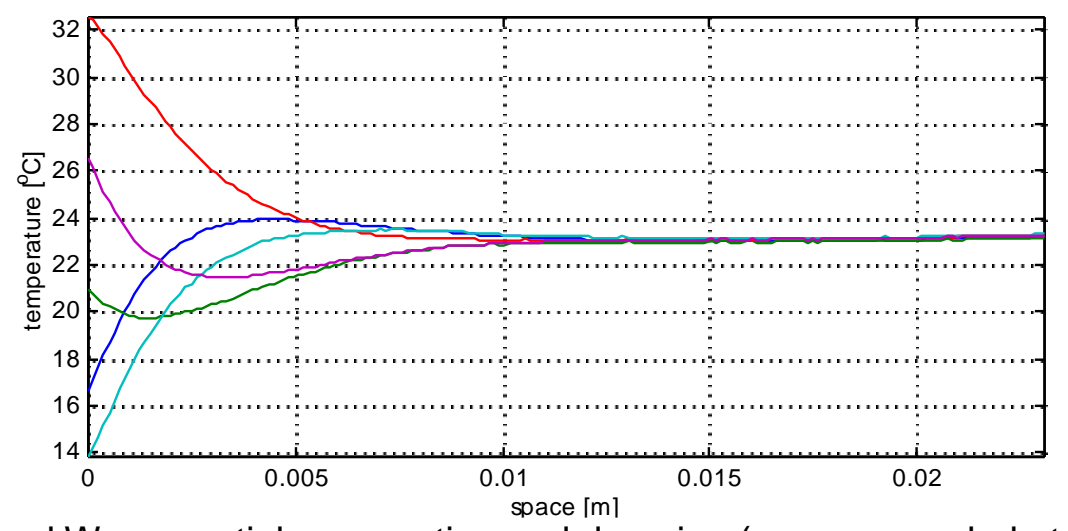

Figure 4c. Thermal Wave spatial propagation and dumping (curves sampled at increasing times) for PVC $1.5 \mathrm{~mm}$ thick and period $\cong 150 \mathrm{~s}$ for the applied harmonic heat flux 


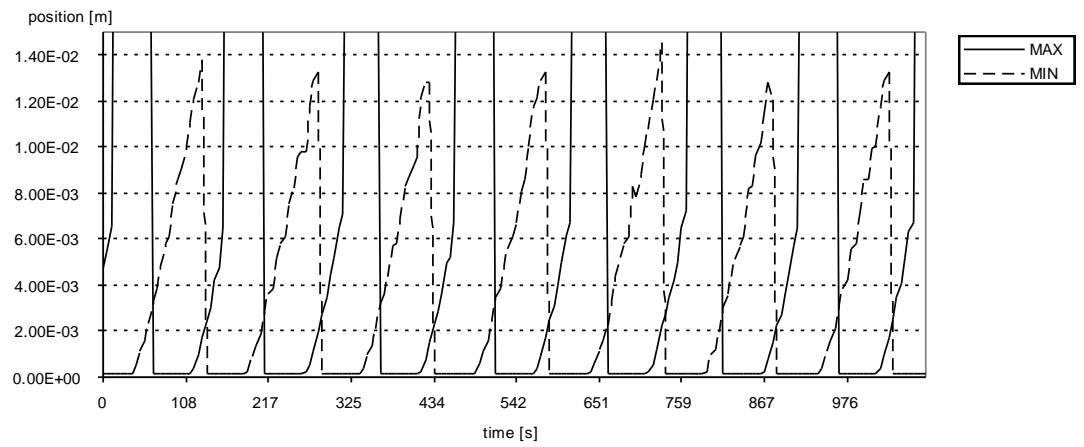

Figure 5a. Space-time plot of maximum and minimum position tracking; slope gives velocity, than diffusivity for PVC $1.5 \mathrm{~mm}$ thick (period $\cong 150 \mathrm{~s}$ )

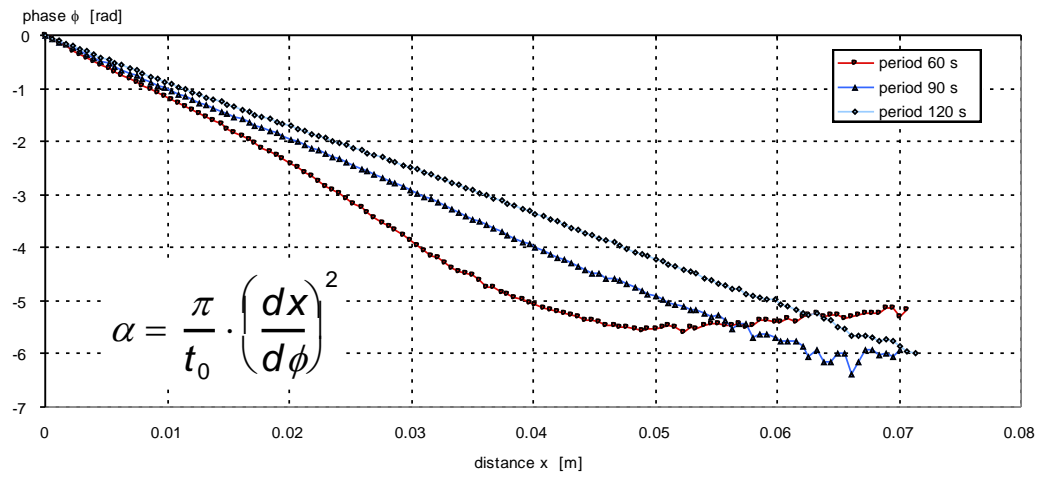

Figure $5 \mathrm{~b}$. example of phase vs space for inconel at 3 different periods

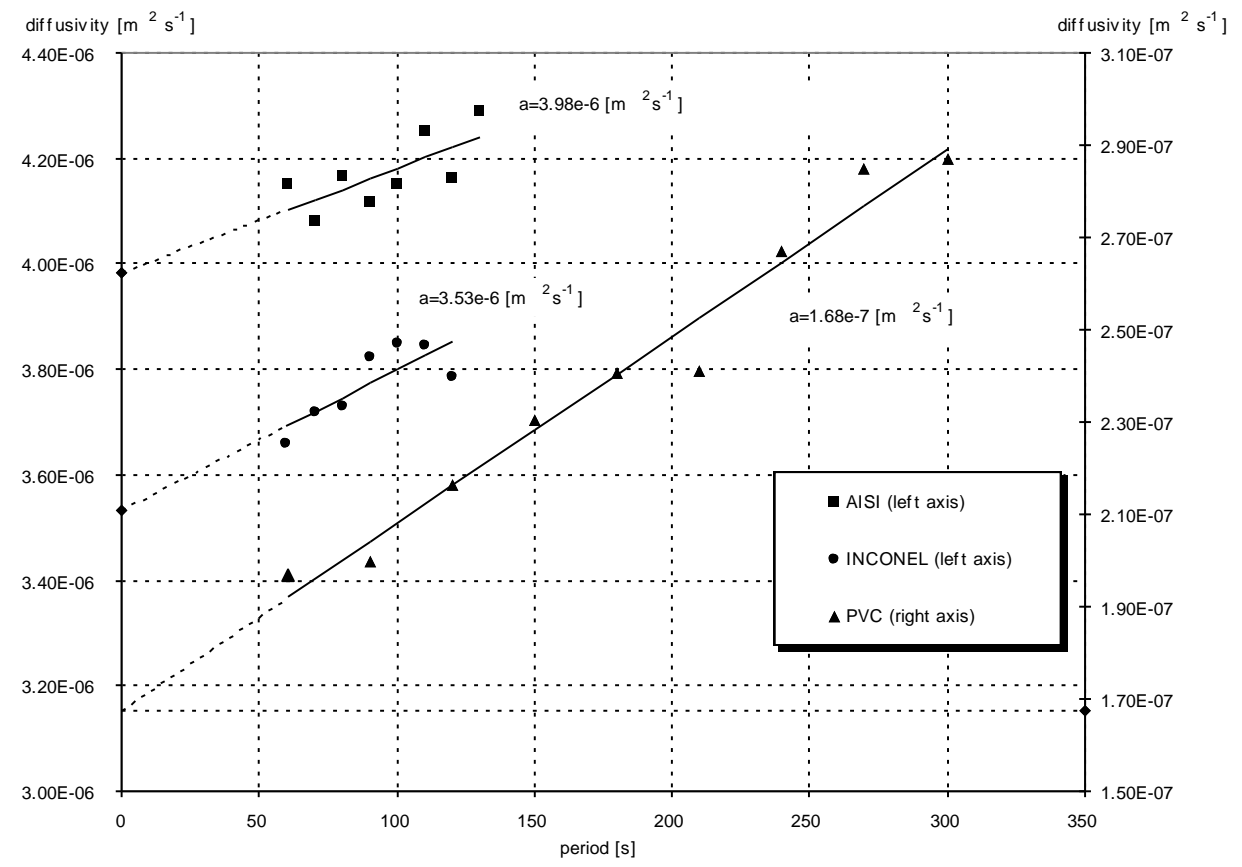

Figure 6. Results of thermal diffusivity measured by LiD for different materials 